\title{
PERFORMANCE OF ENERGY CONSERVATION MODELS, GENERIC, MICAZ AND MICAMOTES, USING AODV ROUTING PROTOCOL ON A WIRELESS SENSOR NETWORK
}

\author{
SURESH KUMAR*1, KIRAN DHULL*, PAYAL ARORA*AND ASHISH KR. LUHACH ${ }^{\dagger}$
}

\begin{abstract}
Wireless Sensor Networks (WSN's) have gained a considerable importance and are used for a variety of applications. In WSN, an arrangement of sensor nodes is done to sense and collect information from its nearby environment and to send it back to the base station using routing protocol. The biggest challenges are how to handle the routing problems and to optimize the energy consumption in WSN. In this paper, performance evaluation of three energy models, Generic, Micaz and Micamotes, is presented using Ad-Hoc On demand Distance Vector (AODV) routing protocol. The performance evaluation is done using several parameters: Throughput, Jitter, Average End-to-End Delay (AEED), Total Packets Received (TPR) and Energy consumption in three modes (transmit, receive and idle). Based on the evaluation, it has been found that Micamotes energy model using AODV routing protocol consumes less energy by $80.46 \%$ and $428.57 \%$ in transmit mode, $102.94 \%$ and $335.6 \%$ in receive mode from Micaz and Generic energy models, respectively.
\end{abstract}

Key words: WSN, AODV, Mobile Ad-hoc Networks (MANETS), Route Reply with Error (RRER), Wireless Personal Area Networks (WPAN), Probabilistic Energy Efficient Routing (PEER), Dynamic MANET On-demand (DYMO), AEED, TPR

AMS subject classifications. 68M10,68M20,60K05, 60K25

1. Introduction. WSN is a group of multi functional sensor nodes used for recording and monitoring the environmental conditions such as sound, pollution level, vibrations, temperature, wind, humidity, seismic events etc. positioned over a geographical area. The architecture of WSN is shown in Figure 1.1. Some applications of WSN are area monitoring, industrial Monitoring, health care monitoring, environment sensing etc. [1].

In WSN, energy can be consumed usefully and wastefully. Hence, to improve the energy efficiency, it is essential to reduce the wastage of energy so that the network can work with better efficiency and more battery lifetime. The main focus of energy efficient protocols is reducing the energy consumption during network activities [2].The protocol design for lifetime-maximization and improvement in performance of lifetime for a WSN is expressed by the average network lifetime. It is defined as the average amount of time until the network expiry [3].

Organization of this paper is as follows. Performance analysis of three energy models Generic, Micaz and Micamotes is done using AODV routing protocol based on several performance metrics. Section 2 gives a brief description of AODV protocol used for simulation and explains several energy models. The related current research work is given in Section 3. Section 4 relates with the simulation model and parameters used for simulation. The results of the simulative model are discussed in Section 5 followed by Conclusion in Section 6 .

\section{Routing Protocols and Energy Models.}

2.1. AODV. AODV is a new level of the destination sequence distance vector routing protocol for MANETS with a different mechanism for routing information. It is purely an on demand protocol. The important feature of AODV is that it is time based working protocol. AODV gives demand and destination sequence number on the basis of latest information for the route to destination. AODV takes less time to set up connection. Due to all these advantages, AODV has become popular nowadays. It works in two steps: path discovery and path maintenance. In the path discovery phase, a connection is established between source and destination nodes using Route Request (RREQ) and Route Reply (RREP) packets as shown in Figure 2.1.

The source node sends a RREQ to the nearest neighbouring nodes. If a destination node is found, it replies back with a RREP otherwise the RREQ propagates to other neighbours. While in the second phase, the nodes which receive RREP provide information to the source node about their routing table for changes in topology. In case of link breakage, RRER is sent to the source node to again initiate the routing process.

\footnotetext{
*Department of Electronics and Communication Engineering, University Institute of Engineering and Technology, Maharshi Dayanand University, Rohtak, Haryana, India (sureshvashist.uiet.ece@mdurohtak.ac.in, kirandhull19@gmail.com, payalarora325@gmail.com ).

${ }^{\dagger}$ The PNG University of Technology, Papua New Guinea (ashishluhach@gmail.com ).
} 


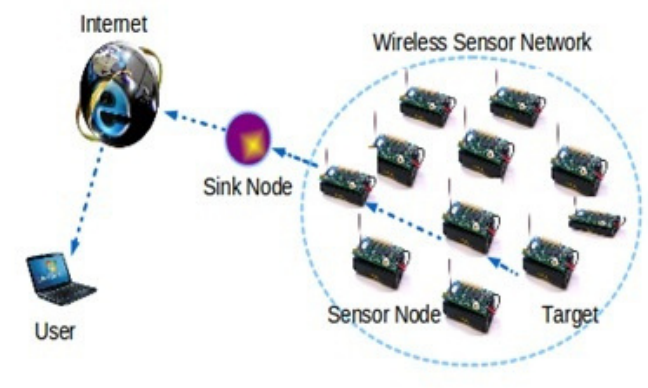

FIG. 1.1. Architecture of WSN

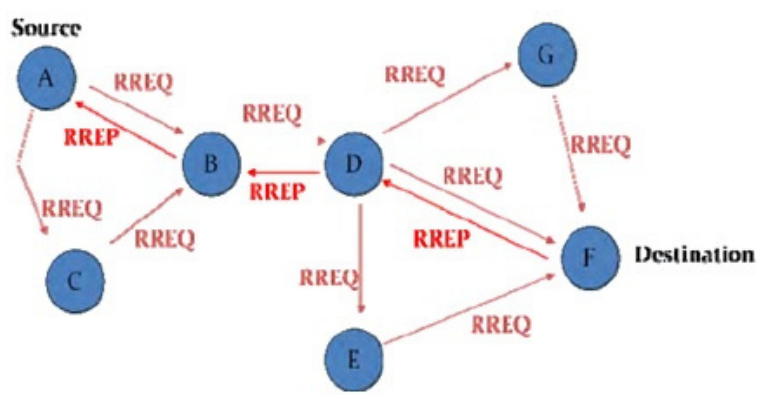

FIG. 2.1. AODV Routing Protocol

2.2. ZigBee. It is a high-level communication protocol based on IEEE 802.15.4 specification used in WPAN. Figure 2.2 shows the ZigBee network. It is simple, inexpensive and consumes less power and data rate than other WPANs. It has several applications such as home energy monitors, wireless light switches and traffic management systems. It limits the transmission distance for low power consumption. It is employed in low data rate applications that involve longer battery life, secure networking and low-latency communication. The topologies used in ZigBee layer are Star, Tree and Generic Mesh. In star network topology, there is one coordinator device which is the central node. The communication network can be extended by the use of ZigBee routers in tree and mesh topology.

2.3. Generic. The Generic model is used for reducing the energy consumption by decreasing the task that have to be done by the sensors and their associated networks. Some methods are used to refill the energy capacity of sensor by building components and mechanisms for additional energy harvesting while operation with the environmental conditions [4].

2.4. Micaz. Micaz is a third generation device used for low power WSNs development. It is an IEEE 802.15.4 standard based RF transceiver which uses2.4 GHz Mote module. It works on 2.4 to $2.48 \mathrm{GHz}$ for deeply embedded sensor network. It is always characterised with larger range and high data rate due to $3 \mathrm{G}$ mote [5].

2.5. Micamotes. Micamotes is a second generation mote broadly used for enabling low power WSNs by developers and researchers. It always runs at $4 \mathrm{MHz}$ and use processor named Atrnel ATmega $128 \mathrm{~L}$ that is 8 bit microcontroller and has $128 \mathrm{~Kb}$ flash memory for storing the mote program. It consumes $8 \mathrm{~A}$ and $15 \mathrm{~A}$ in running mode and sleep mode respectively.Micamotes use two AA batteries that can run for more than a year which generate $1000 \mathrm{~mA}$-hour and has small energy consumption. ATmegacan be operated for about 120 hours at $8 \mathrm{~mA}$. Tiny OS is operating system where Micamotes is built [5].

3. Related and Current Research Work. In [6], a comparative analysis of two energy models (Micamotes and Micaz) and modulation formats has been presented to determine the energy efficiency and Quality 


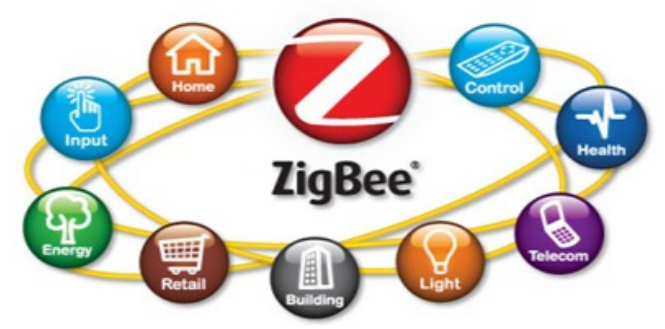

FIG. 2.2. ZigBee Network

of Service (QoS) of WSN. The authors have revealed that the Micamotes energy model performs efficiently with ASK modulation format than BPSK and O-QPSK. In terms of energy efficiency, Micaz energy model consume less energy than Micamotes in all the WSN modes. The ASK modulation is superior in terms of QoS with higher throughput, lower jitter and delay. The BPSK format has small fraction of error of all the three formats. In [7], authors have compared and analysed routing protocols -AODV and DYMO on two mobile CBR network based on parameters like time required for information transmission from sender to receiver, throughput, jitter and AEED. On simulation, it is found that AODV protocol is better than DYMO at same number of nodes and parameters.

In [8], the authors have proposed a hybrid combination of Genetic Algorithm (GA) with Artificial Neural Network (ANN)for energy minimization in a Wireless Mesh Network. Proposed model is compared with AODV and GA based on hop count, energy consumption and lifetime of nodes. It has been observed that the proposed ANN with GA gives better result with energy saving of 51\%. In [9], authors have proposed Modified AODV (M-AODV) protocol to enhance system performance and lifetime of a WSN. The M-AODV protocol has two additional important parameters in HELLO Packets- trust and eagerness of the node which is based on the remaining battery. The simulation results based on Packet Delivery Ratio (PDR), throughput,packets dropped, routing and control overhead indicate that M-AODV is superior compared to AODV protocol.

In [10], the authors have presented a Probabilistic protocol (PEER) to overcome the issue of energy efficiency in a WSN. The overall network's lifetime is improved by balancing the energy consumption and probabilistic forwarding of route requests. On comparison with AODV and Energy Efficient AODV (EEAODV), this protocol shows improvement in battery capacity by $5 \%$. In [11], authors have performed a comparison of DSR and DYMO protocol based on CBR and multi-clustering technique using parameters- throughput, delay, TPR at the PAN coordinator and battery capacity. It was concluded that DYMO performs better in terms of all performance metrics. In [12], a comprehensive review of WSN's has been presented for conservation of energy regarding several design issues such as collection and aggregation of data, clustering and routing based on structure free and structured modelling. From the study of routing protocols, it has been found that a routing protocol is best saving if it minimizes number of data transmissions, the total energy spent and maximizes the number of alive nodes in WSN.

In [13], the authors have presented a review of routing protocols used in Adhoc WSN. It also revealed that the use of ZigBee application with WPAN are highly reliable in mesh networks with many advantages such as lesser cost, increased range, longer battery life and low power consumption.

In [14], the authors have done a comparative analysis of AODV and DSR based on several QoS metrics in AWSN using Zigbee. The AODV routing protocol is found better than DSR for all metrics which therby enhances the QoS.

In [15], the performance of various Ad-Hoc routing protocols (Table Driven- Intrazone Routing Protocol (IARP), On Demand- Interzone Routing Protocol (IERP), Dynamic Source Routing (DSR) and Hybrid- Zone Routing Protocol (ZRP))has been analysed in terms of different performance metrics. It was revealed from the results that IERP and DSR have better efficiency for 3D terrestrial data communication. Also, the authors have suggested that in future, field testing can be used for detecting errors in data. In [16], Mobility Aware and Dual Phase AODV with Adaptive Hello Messages routing protocol is proposed which is an extension of the 


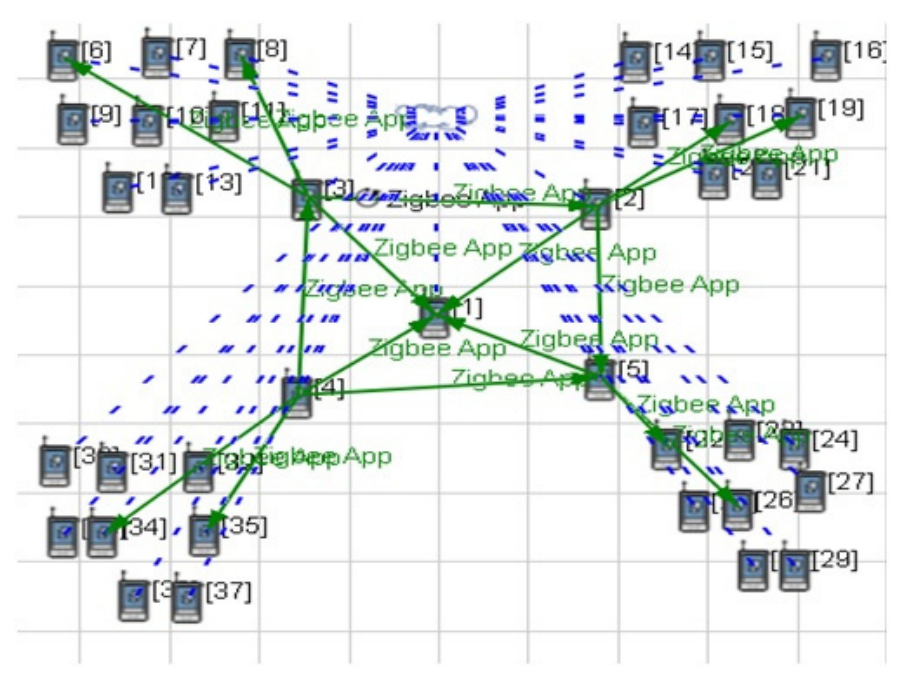

FIG. 4.1. The scenario architecture

AODV protocol. This proposed protocol is found efficient for route discovery procedure which thereby helps in finding more stable routes compared to conventional AODV protocol.

In [17], authors have proposed a fuzzy controlled rate and Hello interval based congestion control which on the basis of energy consumed and node mobility changes the interval of HELLO packets. An improvement in performance parameters is found when the HELLO messages are made adaptive. It has been concluded that the frequency of HELLO messages can be modified to control congestion. In [18], authors have evaluated the performance of protocols- AODV, ZRP and DSR to control congestion in MANET's in terms of error packets, collided packets, throughput and number of transmitted packets for different number of nodes. The simulative results indicate that the AODV routing protocol is effective in controlling congestion.

In [19], an algorithm for cluster head selection has been proposed with the LEACH clustering protocol based on distance, residual energy and reliability. From the observation, it has been analysed that proposed algorithm is more efficient, stable and enhances the lifetime of the network by balancing of nodes and efficient information delivery. So far, the researchers have not evaluated the sensor nodes for all the parameters i.e.Throughput, Jitter, AEED, TPR and Energy consumption in all three modes (transmit, receive and idle) together which has motivated us to do this.

4. Simulation Model. In this work, simulation and analysis is done using QualNet 7.3.1 network simulator.The designed network consists of 37 nodes that are placed randomly in the $1000 \mathrm{~m} \mathrm{X} \mathrm{1000m} \mathrm{terrain} \mathrm{where}$ node 1 act as a sink node which is Full Function Device (FFD) and rest are Reduced Function Devices (RFD). The scenario architecture is shown in Figure 4.1. There are 100 packets of data transmitted with each packet containing about 70 bytes of data. Node 1 is connected to nodes $2,3,4$ and 5 via wireless link in a star topology that receives the data from central PAN co-ordinator. Some nodes are not involved in the process of communication. ZigBee applications are only applied between nodes 1, 2, 3, 5, 8, 18, 19, 22 and 35. The performance of various energy models such as Generic, Micaz and Micamotes are analysed on the basis of network metrics such as Throughput, Jitter, AEED, TPR and Energy Consumption in all the nodes using AODV routing protocol. The parameters used for simulation are given in Table 4.1.

5. Results and Discussion. In this proposed work, we have simulated the designed network using AODV routing protocol in order to analyze the performance of three energy models i.e. Generic, Micaz and Micamotes. Five performance parameters are taken into consideration which is explained one by one in the following section:

5.1. Throughput. It is defined as the total data arrived at the receiver through network in a given time and always taken in bits/ seconds. The analysis of average throughput obtained in case of three energy models of the proposed WSN is depicted by the Figure 5.1. On analysis of above plotted graph, it can be concluded that 
TABLE 4.1

Scenario Parameters

\begin{tabular}{|c|c|}
\hline Parameter & Value \\
\hline Simulator & Qualnet 7.3 .1 \\
\hline Terrain Size & $1000 \mathrm{~m}^{*} 1000 \mathrm{~m}$ \\
\hline No. of Nodes & 37 \\
\hline MAC Protocol & IEEE 802.15 .4 \\
\hline Packet Reception Model & PHY 802.15 .4 \\
\hline Radio Type & IEEE 802.15 .4 \\
\hline Energy Model & Generic, Micaz, Micamotes \\
\hline Routing Protocol & Amnidirectional \\
\hline Antenna Model & IPV4 \\
\hline Network Protocol & Sensor(RFD, FFD) \\
\hline Device Type & ZIGBEE \\
\hline Traffic Type & 100 \\
\hline Items to Send & 70 Bytes \\
\hline Item Size & 500 secs \\
\hline Simulation time & Wireless \\
\hline Link & 868MHz \\
\hline Channel Frequency & O-QPSK \\
\hline Modulation &
\end{tabular}

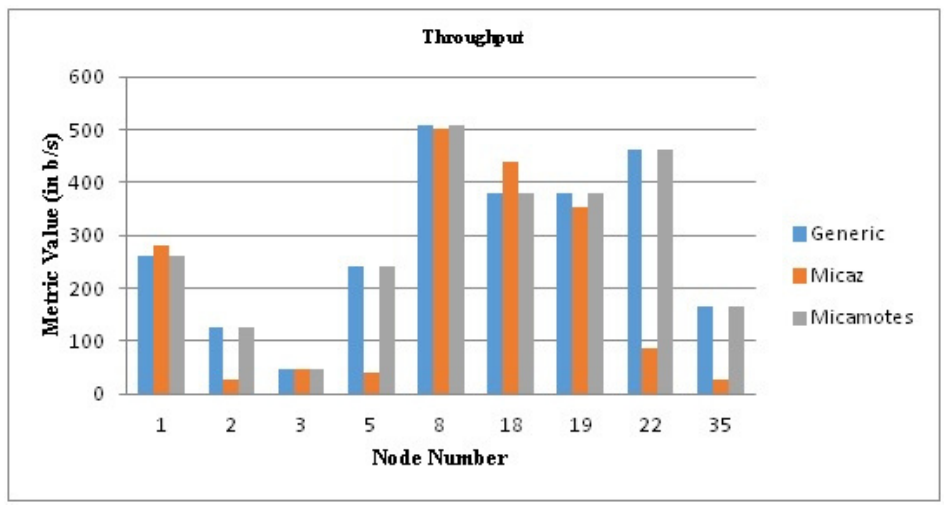

FIG. 5.1. Throughput for different Energy Models

maximum throughput is obtained for Generic and Micamotes energy models because of their reactive nature and least in case of Micazas it is hybrid in nature depending upon number of participating nodes. Therefore, on the basis of data packets received, Generic and Micaz gives better performance at the destination per unit time.

5.2. AEED. It is defined as average time required to send a data packet to destination. It is also known as data latency and is measured in seconds. Analysis between three models on the basis of data latency for the proposed WSN is shown in the Figure 5.2 given below. On analyzing the above plotted graph, it is found that the Micaz has smaller AEED because of its reactive nature compared to others which are proactive in nature. Therefore, the instant at which the first packet will receive is lesser in case of Micaz as compared to Generic and Micamotes.

5.3. Jitter. It is defined as the variation in the latency of packets at the receiver due to congestion, topology, route change etc. It is measured in seconds.It arises when the packets takes different amount of time, 


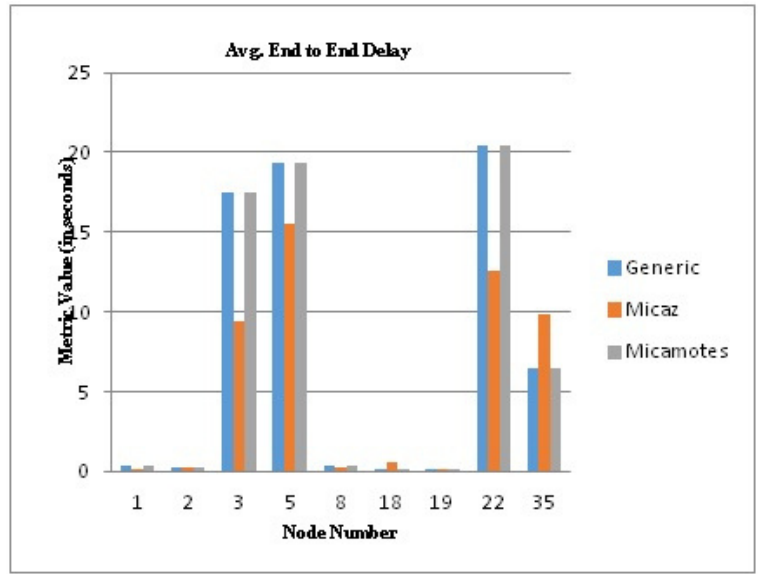

FIG. 5.2. AEED for different Energy Models

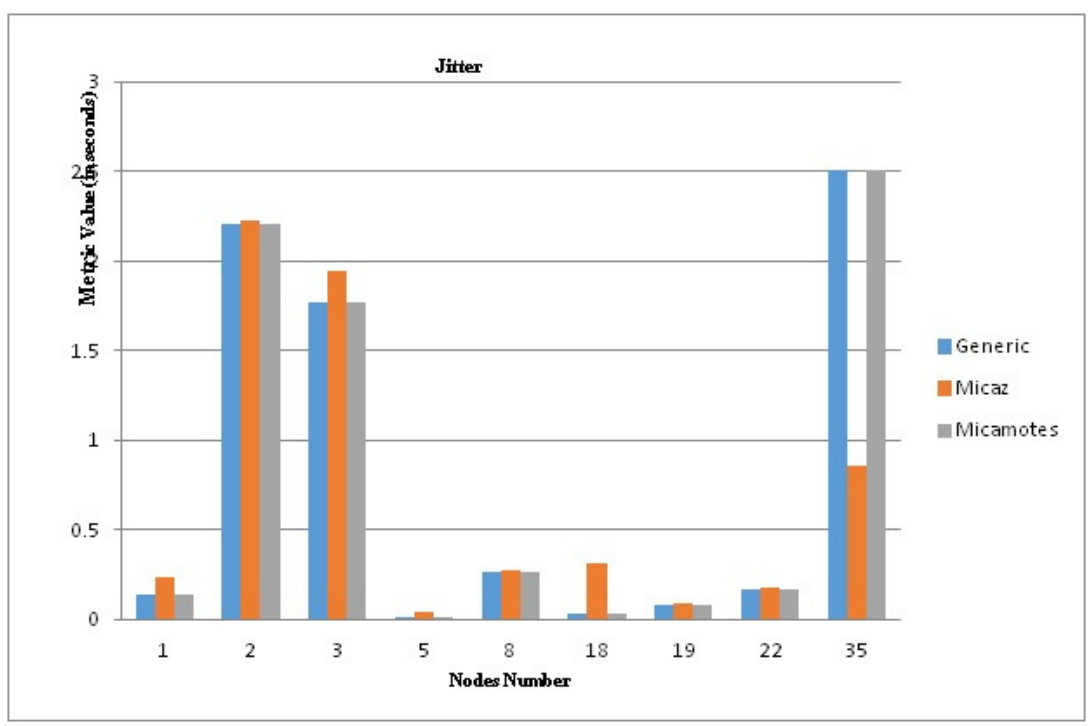

FIG. 5.3. Jitter for different Energy Models

reaching from source to destination in a transmission scenario. For the better performance, its value should be as low as possible. The analysis of jitter obtained in case of three energy models of the proposed WSN is depicted by the Figure 5.3. On observing the graph, it is noted that jitter is maximum for Micaz which in proactive in nature while smaller for the rest which are reactive in nature. Proactive models shows maximum jitter and reactive shows smaller jitter.

5.4. TPR. It determines the overall message received successfully at destination by any server. Efficiency of the network will be high, if the number of message received per unit time is more. Figure 5.4 shows the graph of total message received. It has been analysed from the figure that there are least number of packets received in case of Micaz which is proactive in nature while packets received are higher forrest of two which are reactive in nature. To enhance reliability of energy model, the network must receive higher number of packets. Hence, the reactive protocol provides good results.

5.5. Energy Consumption. The WSN parameters including lifetime, response time, scalability and effective sampling frequency etc. depends upon the power. To preserve the health of each node, energy is required 


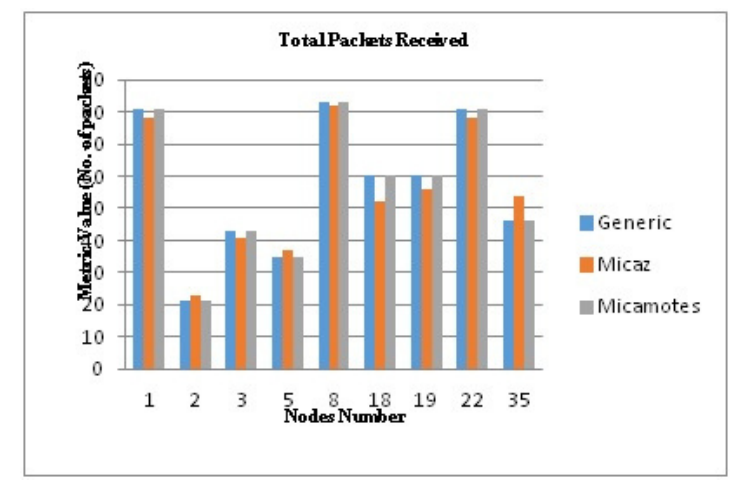

FIG. 5.4. TPR for different Energy Models

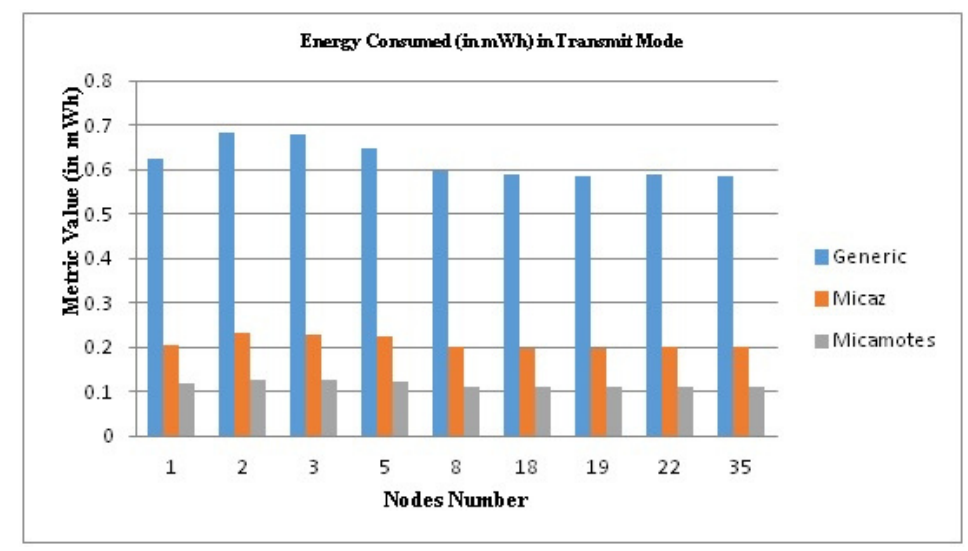

FIG. 5.5. Energy Consumed for different Energy Models in Transmit Mode

during transmission and reception of the data packets. The Figure 5.5 represents the energy consumption in transmit mode for various energy models i.e. Generic, Micaz and Micamotes. It has been observed from the graph that Generic model which is proactive in nature consumes more energy while the Micaz which is hybrid in nature operates in moderate energy and Micamotes which is reactive in nature requires lesser energy. Hence, Micamotes model is more energy efficient in comparison with Generic model. The Figure 5.6 shows energy consumed in Receive mode that is similar results as obtained for the energy consumed in case of transmit mode by routing protocol named AODV.

From analysis, it has been found that Generic Model which is proactive in nature consumes highest energy while Micamotes which is reactive in nature requires least energy. Therefore, Micamotes model prove to be more energy saving than Generic and Micaz models. The energy consumed by different models in idle mode is depicted in Figure 5.7. It has been observed that idle mode shows different results in comparison to transmit and receive modes.

In case of idle mode, the energy consumption is comparatively higher in Generic than other two modes. Therefore, Micamotes seems to be best energy saving model than other two models. It is also noted that for all the energy models, most of the energy consumption occurs in the idle mode.

After simulating, it has been observed that the energy consumption is highest in case of Generic model and lowest in case of Micamotes energy model. Therefore, Micamotes has been proved to be the best energy efficient model as compared to Generic and Micaz energy models in transmit, received and idle modes. The sensor networks can also be analyzed using other routing protocols for different design layouts and simulation parameters. 


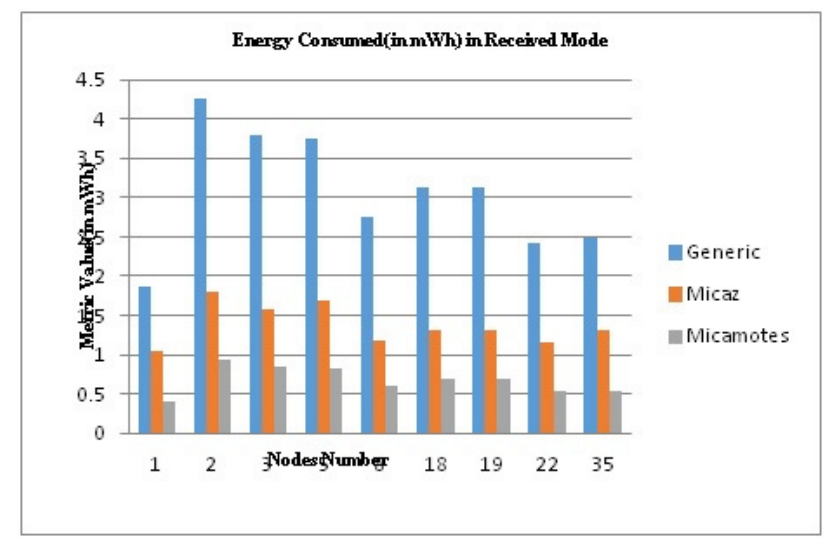

FIG. 5.6. Energy Consumed for different Energy Models in received mode

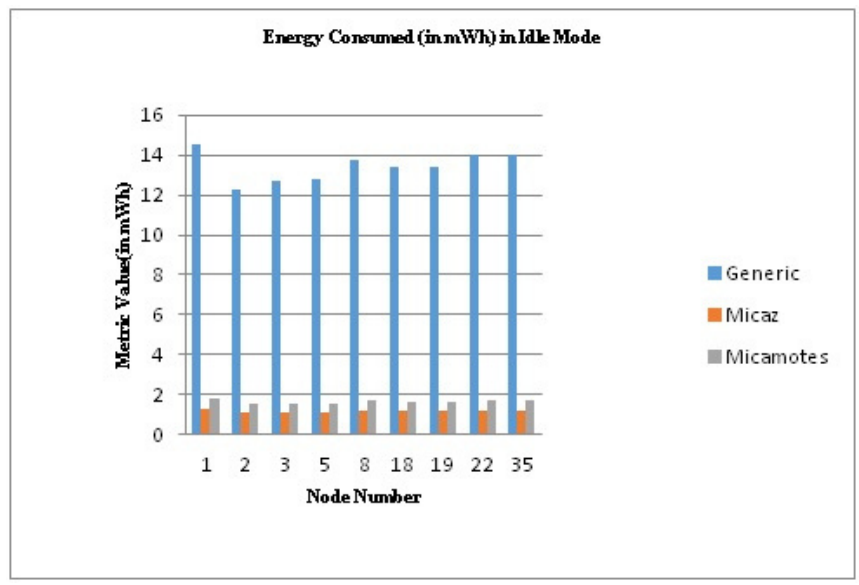

FIG. 5.7. Energy Consumed for different Energy Models in idle mode

6. Conclusion. This paper gives performance analysis of three energy models i.e. Generic, Micaz and Micamotes using AODV routing protocol on the QualNet simulator and their performance analysis is done on the basis of network metric such as Throughput, Jitter, AEED, TPR, and Energy Consumption in all the nodes. From simulation results it is concluded that Micaz has least throughput and maximum average jitter compare to Generic and Micamotes. AEED and TPR are least for Micaz and similar in Generic and Micamotes. For energy consumption, Micamotes consumes less energy by $80.46 \%$ and $428.57 \%$ in transmit mode, $102.94 \%$ and $335.6 \%$ in receive mode from Micaz and Generic energy models respectively. However, in idle mode Micaz energy model is found to be consuming less energy by $54.36 \%$ and $23.45 \%$ than Micamotes and Generic energy models respectively. Therefore, in over all analysis Micamotes seems to be the best energy saving model in all modes using AODV routing protocol.

\section{REFERENCES}

[1] C. Karthik, S. Kumar, R.S. Kumar, M. Nageswari, Sensors Lifetime Enhancement Techniques in Wireless Sensor Networks - A Critical ReviewInternational Journal of Computer Science and Information Technology Security, Vol. 3, April 2013.

[2] Y. Yu, B. Krishnamachari, V.K Prasanna, Issues in Designing Middleware for Wireless Sensor Network, IEEE Network, Vol.18, 2014, pp 15-21.

[3] Y. Chen, Q. ZhaO, On the Lifetime of Wireless Sensor Networks, IEEE Communications Letters, Vol. 9, November 2005.

[4] A. Goyal, S. Vijay, D. Kumar, Simulation and Performance Analysis of Routing Protocols in Wireless Sensor Network 
using QualNet, International Journal of Computer Applications, (ISSN 0975-8887) Vol. 52, August 2012, pp 47-50.

[5] N. Kamyabpou, D.B. Hoang, LU-Modeling Overall Energy Consumption in Wireless Sensor Networks,2007.

[6] S.K. Singh, R. Paulus, S.V. Rajput, T. Kaur, Analysis of energy model and QoS in wireless sensor network under different modulation schemes, International Conference for Convergence of Technology, (ISSN 4799-3759) Vol. 1, 2014.

[7] N. Radhey, V. NAndal, Simulation and Analysis of AODV and DYMO Protocols under CBR in Wireless Sensor Network using QualNet,International Journal of Computer Sciences and Engineering, (ISSN 2347-2693) Vol. 6, May 2018, pp 100-104.

[8] B. Prakash, S. Jayashri, T.S. KARThik, A hybrid genetic artificial neural network (G-ANN) algorithm for optimization of energy component in a wireless mesh network toward green computing, Soft Computing, (ISSN01234567).January 2019. doi: 10.1007/s00500-019-03789-8.

[9] H. K. Sampada, K. R. Shobha, Performance Analysis of Energy-Efficient MANETs-Using Modified AODV (M-AODV), ICCNCT 2019, doi: 10.1007/978-981-10-8681-6_9, pp 75-86

[10] M, Rajgor, P. Shete, R.N. Awale, Probabilistic Energy Efficient Routing ProtocoL, International Conference on Communication", Information and Computing Technology. Vol. 1, 2018.

[11] Muhammad A.K. Pandey, P.N. Gupta, H. Vardhan, Performance Evaluation of Various Routing Protocols and quality of service for Wireless Sensor Network, Journal of Telecommunication Study, vol .4

[12] S. YADAV, R.S YADAV, A review on energy efficient protocols in wireless sensor networks, Wireless Network, 2018, DOI :10.1007/s11276-015-1025-X.

[13] K. Mor, S. Kumar, D. Sharma, Ad-Hoc Wireless Sensor Network Based on IEEE 802.15.4: Theoretical Review, International Journal of Computer Sciences and Engineering" (ISSN:2347-2693) Vol. 6, 2018, DOI: https://doi.org/10.26438/ijcse/v6i3.220225 pp.220-225

[14] K. Mor, S. Kumar, Evaluation of QoS Metrics in Ad-Hoc Wireless Sensor Networks using Zigbee, International Journal of Computer Sciences and Engineering", (ISSN 2347-2693) Vol. 6, March 2018, DOI: 10.26438/ijcse/v6i3.9296 pp.92-96.

[15] R. Monir, R. Thalore, P. P. Bhattacharyana, Performance Comparison and Analysis of On-Demand, Table-driven and Hybrid Routing Protocols in 3D, Mody University International Journal of Computing and Engineering Research, (ISSN 2456-9607) Vol.2, 2018, pp 20-25.

[16] K.A. Darabkh, M.S.E Judeh, An Improved Reactive Routing Protocol over Mobile Adhoc Networks,2018, pp 707-711.

[17] N. Kaur, R. Singhai, Minimizing Congestion in Mobile Ad hoc Network Using Adaptive Control Packet Frequency and Data Rate, Proceedings of ICCASP, 2018, DOI:.org/10.1007/978-981-13-1513-8_30 pp 285-294.

[18] S. Khurana, S. Kumar, D. Sharma, Performance Evaluation of Congestion Control in MANETs using AODV, DSR and ZRP Protocols, International Journals of Advanced Research in Computer Science and Software Engineering, (ISSN: 2277-128X) Vol. 7, June 2017, pp 398-403.

[19] R. Munjal, S. Kumar, Maximizing Lifetime of Cluster-based WSN through Energy-Efficient Clustering Method, International Journal of Mobile and Ad-hoc Network, Vol. 2, August 2011, pp 239-243.

Edited by: Dharm Singh Jat

Received: May 22, 2019

Accepted: Nov 14, 2019 